\title{
Erratum to: Block-Sobolev Spaces and the Rate of Almost Everywhere Convergence of Bochner-Riesz Means
}

\author{
Dashan Fan' ${ }^{1}$ Fayou Zhao
}

\section{Erratum to: Constr Approx DOI 10.1007/s00365-016-9343-5}

We corrected three errors in our article.

1. Page 2, line -11, "proved" should be "conjectured".

2. Page 2, line -5 , "result" should be "conjecture".

3. Page 5, line -4, " $\phi_{0}, \phi_{1}, \phi_{\infty} \in \mathscr{S}\left(\mathbb{R}^{n}\right)$ " should be " $\phi_{0}, \phi_{1} \in \mathscr{S}\left(\mathbb{R}^{n}\right)$ ".

The online version of the original article can be found under doi:10.1007/s00365-016-9343-5.

$\triangle$ Fayou Zhao

fyzhao@shu.edu.cn

1 Department of Mathematical Sciences, University of Wisconsin-Milwaukee, Milwaukee, WI 53201, USA

2 Department of Mathematics, Shanghai University, Shanghai 200444, People's Republic of China 